\title{
A Study on the Reduction Behavior of FeO by Analyzing Pore Characteristics Using the Labyrinth Coefficient at High Temperature
}

\author{
Sang Gyun Shin $(\mathbb{D}$ and Dong Joon Min *(D) \\ Department of Material Science and Engineering, Yonsei University, 50 Yonsei-Ro, Seodaemun-Gu, \\ Seoul 038722, Korea; mang2ca@gmail.com \\ * Correspondence: chemical@yonsei.ac.kr; Tel.: +82-2-2123-2840
}

Citation: Shin, S.G.; Min, D.J. A Study on the Reduction Behavior of FeO by Analyzing Pore Characteristics Using the Labyrinth Coefficient at High Temperature. Metals 2021, 11, 414. https:// doi.org/10.3390/met11030414

Academic Editor: Alexander McLean

Received: 29 January 2021

Accepted: 28 February 2021

Published: 3 March 2021

Publisher's Note: MDPI stays neutral with regard to jurisdictional claims in published maps and institutional affiliations.

Copyright: (c) 2021 by the authors. Licensee MDPI, Basel, Switzerland. This article is an open access article distributed under the terms and conditions of the Creative Commons Attribution (CC BY) license (https:// creativecommons.org/licenses/by/ $4.0 /)$.

\begin{abstract}
The effect of extrinsic porosity on the reduction behavior of $\mathrm{FeO}$ was evaluated by thermogravimetric analysis (TGA), scanning electron microscopy (SEM) and the Brunauer-Emmett-Teller (BET) technique and analyzed using the labyrinth coefficient of FeO. The extrinsic pore exhibited an abnormal effect on reduction behavior in the range of less than $50 \%$ reduction degree, despite the increase in apparent porosity. SEM and BET analysis indicated that the abnormal reduction behavior by extrinsic pores at the initial reduction stage was speculated to be due to the characteristic of extrinsic pore that is open only at one end. However, the overall porosity and reduction rate after a $40 \%$ reduction revert to the normal relationship. In addition, the experimental results indicated that the abnormal effect of the extrinsic pores in the initial stage was mitigated by an increase in the temperature. The abnormal effect of extrinsic porosity on $\mathrm{FeO}$ reduction was mathematically analyzed using the labyrinth coefficient. It can be summarized that not only the number of pores, but also their quality and distribution are important in determining the reduction rate.
\end{abstract}

Keywords: $\mathrm{FeO} ; \mathrm{FeCl}_{2}$; reduction; morphology; porosity

\section{Introduction}

It has been generally accepted that the reduction rate of iron oxide strongly depends on its chemical and physical characteristics during the reduction process. The chemical potential is typically defined by the temperature, oxygen potential, and activity of iron oxide in an ore. Many researchers [1-10] have generally reported that the reduction rate of $\mathrm{FeO}$ increases as the temperature increases and the oxygen potential decreases. In addition, El-Geassy and many other researchers [10-15] have reported that when iron oxide reacts with other oxides to form different phases (magnesioferrite, dolomite, hercynite, SFCA, and jacobsite), the activity of the iron oxide decreases, and consequently, it has a negative effect on reduction. As such, the chemical potential for the reduction of iron oxide has been studied both qualitatively and quantitatively.

Many studies have been conducted on the effect of pores, a major physical property of iron oxide. Huang et al. [16] and Turkdogan et al. [17] studied the effect of porosity change on the reduction rate during the reduction process. The porosity of the pellets during the reduction process was increased owing to the cracks and morphology changes of iron oxide, and the pore size increased with increasing reduction temperature. Matthew et al. [18] reported the morphology and pore shape of reduced iron according to the reduction condition.

There are many studies on the relationship between the reduction of iron oxide and the porosity of the initial state of iron oxide. Bahgat et al. [19] and Kim et al. [20] reported that $\mathrm{SiO}_{2}$ reacts with $\mathrm{FeO}$ to form a dense phase (silicates, fayalite) at equilibrium, thereby reducing the porosity of $\mathrm{FeO}-\mathrm{SiO}_{2}$ pellets. Kim et al. [20] and Takahashi et al. [21] changed the initial state of porosity using $\mathrm{CaO}$, and the corresponding reduction behavior 
was studied. When $\mathrm{CaO}$ is added, the porosity of the $\mathrm{FeO}-\mathrm{CaO}$ pellet increases with its solubility. However, when the solubility increases (about $2.5 \mathrm{wt} \% \mathrm{CaO}$ ), the equilibrium phase forms calcium ferrite and the porosity of $\mathrm{FeO}-\mathrm{CaO}$ decreases, as in the case of $\mathrm{SiO}_{2}$ addition.

Kaneko [22] proposed that the pores can be classified into intrinsic and extrinsic pores with respect to their origin and structure. Intrinsic pores are inherently possessed by the intraparticle arrangement. Extrinsic pores are created during the removal of foreign substances from the base material through various reactions. However, some studies [16-18] only measured the change in intrinsic pores during the reduction process of iron oxide, and there is a limit to extrinsic pore control. In other studies [19-21], pore control was performed through the addition of other substances; however, it is difficult to confirm the effect of pores because the activity of iron oxide is simultaneously changed.

According to Rouquerol et al. [23], pores are classified into closed pores and open pores based their accessibility by to an external fluid. In detail, open pores are again divided into open only at one end (dead-end or saccafe) and open at two ends (through pores). Among them, closed pores and open only at one end (dead-end) adversely affect reduction because the gas flow is not smooth. Meanwhile, the pores that are open at two ends (through pores) help in the reduction process due to the permeability of the gas. The results of previous studies indicated the necessary for discussion of such pore quality.

Therefore, the present investigation was carried out to understand the effects of porosity on the reduction rate of $\mathrm{FeO}$ by introducing extrinsic pores and taking advantage of the sublimation property of $\mathrm{FeCl}_{2}$. $\mathrm{FeCl}_{2}$ has a high vapor pressure at high temperatures. The evaporation behavior of $\mathrm{FeCl}_{2}$ at high temperatures enables the formation of extrinsic pores in $\mathrm{FeO}$ without changing the activity of $\mathrm{FeO}$. Through extrinsic pore control, the effect of the pore alone on reduction can be confirmed. In addition, for better understanding, the porosity and morphological changes (pore quality) are considered together using the labyrinth coefficient, which accounts for the porosity and tortuosity during the reduction process.

\section{Materials and Methods}

\subsection{Sample Preparation}

FeO powder (GR grade, Junsei, Tokyo, Japan) was crushed to a particle size of less than $100 \mu \mathrm{m}$ and pressed in a cylindrical mold under a pressure of $6370 \mathrm{kgf} / \mathrm{cm}^{2}$. Standard $\mathrm{FeO}$ pellets were prepared after removing internal moisture for $24 \mathrm{~h}$ in an oven at $383 \mathrm{~K} . \mathrm{FeCl}_{2}$ (99.99\%, Sigma-Aldrich, St.Louis, MI, USA) and standard FeO were crushed together to a particle size of less than $100 \mu \mathrm{m}$. The obtained powder mixture was pressed in a cylindrical mold under a pressure of $6370 \mathrm{kgf} / \mathrm{cm}^{2}$. Table 1 shows the chemical compositions of the samples. $\mathrm{FeCl}_{2}$ has high vapor pressure at high temperatures [24], for example, $0.09 \mathrm{~atm}$ at $1073 \mathrm{~K}, 0.3 \mathrm{~atm}$ at $1173 \mathrm{~K}$, and approximately $0.93 \mathrm{~atm}$ at $1273 \mathrm{~K}$. The prepared pellets were charged into a vertical tube furnace, set at a temperature corresponding to the reduction temperature, and $\mathrm{FeCl}_{2}$ was evaporated in $\mathrm{FeO}$ under a high-purity $\mathrm{Ar}$ gas $(99.9999 \%)$ for $1 \mathrm{~h}$. Extrinsic pores are produced at the site where $\mathrm{FeCl}_{2}$ is evaporated. The samples were quenched in an Ar atmosphere. Table 1 also shows the initial contents of $\mathrm{FeCl}_{2}$ and the corresponding $\mathrm{Cl}$ contents after heating at different temperatures. The amount of residual $\mathrm{Cl}$ present in the sample was analyzed using combustion ion chromatography (CIC, AQF100, 881 Compact IC pro, Metrohm, Herisau, Switzerland). The amount of residual $\mathrm{Cl}$ in the sample was less than $350 \mathrm{ppm}$, except for the case of $9 \mathrm{wt} \% \mathrm{FeCl}_{2}$ (1836 ppm) evaporated at $1073 \mathrm{~K}$. It is assumed that there is no change in the chemical potential of $\mathrm{FeO}$ due to the presence of residual $\mathrm{FeCl}_{2}$ in the reduction process. Figure 1 shows the results of the analysis of FeO morphology by SEM (SM-7001F, JEOL) after extrinsic pore formation with $\mathrm{FeCl}_{2}$ at $1173 \mathrm{~K}$ before reduction. Before (Figure 1a) and after (Figure $1 \mathrm{~b}-\mathrm{d}$ ) extrinsic pore formation, there was a difference in morphology, and it was judged that the quality of the pores changed accordingly. 
Table 1. Chemical compositions of the sample and the corresponding $\mathrm{Cl}$ contents after heating at different temperatures.

\begin{tabular}{|c|c|c|c|c|c|}
\hline $\begin{array}{l}\text { Sample } \\
\text { Name }\end{array}$ & $\mathrm{FeO}(\mathrm{g})$ & $\mathrm{FeCl}_{2}(\mathrm{~g})$ & $\begin{array}{c}\text { Initial } \mathrm{FeCl}_{2} \\
\text { Contents }\left(\mathrm{Cl}_{2}\right. \\
\text { Contents) }(w \mathrm{w} \%)\end{array}$ & $\begin{array}{c}\text { Temperature } \\
\text { (K) }\end{array}$ & $\begin{array}{c}\text { Residual Cl } \\
\text { Contents } \\
\text { (ppm) }\end{array}$ \\
\hline $0 \mathrm{FeCl}_{2}$ & 1.00 & - & - & - & - \\
\hline $2 \mathrm{FeCl}_{2}$ & 0.98 & 0.02 & $2(1.12)$ & $\begin{array}{l}1073 \\
1173 \\
1273 \\
\end{array}$ & $\begin{array}{c}170 \\
93 \\
70\end{array}$ \\
\hline $5 \mathrm{FeCl}_{2}$ & 0.95 & 0.05 & $5(2.80)$ & $\begin{array}{l}1073 \\
1173 \\
1273\end{array}$ & $\begin{array}{l}336 \\
232 \\
183\end{array}$ \\
\hline $9 \mathrm{FeCl}_{2}$ & 0.91 & 0.09 & $9(5.03)$ & $\begin{array}{l}1073 \\
1173 \\
1273\end{array}$ & $\begin{array}{c}1836 \\
332 \\
315\end{array}$ \\
\hline
\end{tabular}
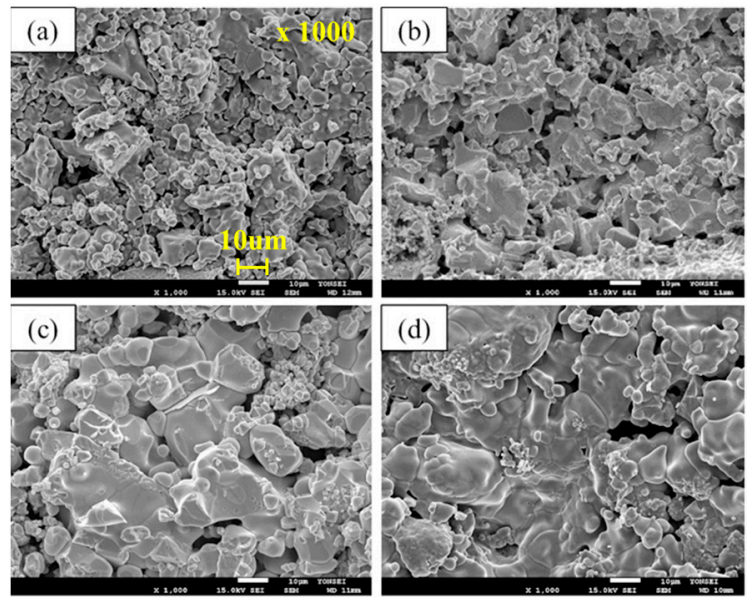

Figure 1. SEM micrographs of FeO sample with different initial porosity in FeO, obtained at $1173 \mathrm{~K}$, under inert gas condition; Initial overall porosity in $\mathrm{FeO}$ (a) 23.9, (b) 26.6, (c) 31.6, and (d) 35.5.

\subsection{Analysis of Reduction Behavior}

The sample was charged into a thermogravimetric analyzer (TGA, SETSYS Evolution, Setaram, Caluire-et-Cuire, France), as shown in Figure 2, and then heated to a target temperature in a high-purity $\operatorname{Ar}(99.999 \%)$ atmosphere. When the target temperature was achieved, the Ar gas was replaced with CO gas (99.99\%), and the weight changes were measured every second using TGA. The reduction was carried out in the temperature range of 1073-1273 K. Based on the experimental data obtained through TGA, the reduction rate (R) can be defined as follows:

$$
\mathrm{R}(\%)=\frac{\Delta W_{O}^{t}}{W_{O}^{i}} \times 100
$$

where $W_{O}^{i}$ is the amount of removable oxygen in the initial sample and $\Delta W_{O}^{t}$ is the weight of oxygen removed at time t. The porosity of the sample before and after the reaction was analyzed using a mercury porosimeter (PM33GT, Quamtachrome, Graz, Austria), and the morphology was analyzed by SEM (SM-7001F, JEOL, Tokyo, Japan). 


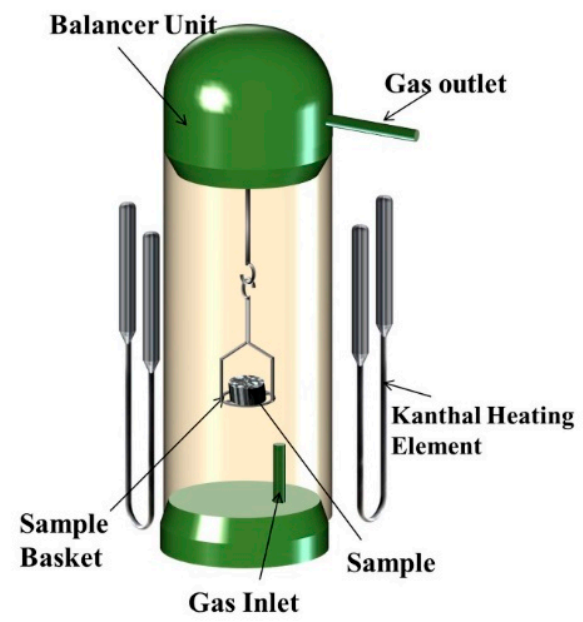

Figure 2. Schematic diagram of the thermogravimetry analyzer (TGA).

\section{Results and Discussion}

The apparent porosity of the initial state of the sample is shown in Table 2. Standard $\mathrm{FeO}$ has a $23.9 \%$ (intrinsic pore) volumetric porosity; however, the extrinsic pore introduced by $\mathrm{FeO}$ using the evaporation of $\mathrm{FeCl}_{2}$ has an overall volumetric porosity of up to $36.4 \%$. As the amount of $\mathrm{FeCl}_{2}$ removed by evaporation increases and the temperature increases, the amount of the introduced extrinsic pores increases. Herisau

Table 2. Porosity change in $\mathrm{FeO}$ sample before the reduction with different initial contents of $\mathrm{FeCl}_{2}$ and different temperatures.

\begin{tabular}{cccccc}
\hline $\begin{array}{c}\text { Initial Cl } \\
\text { Contents } \\
(\mathbf{w t} \%)\end{array}$ & $\mathbf{1 0 7 3 ~ \mathbf { ~ }}$ & $\mathbf{1 1 2 3} \mathbf{K}$ & $\mathbf{1 1 7 3 ~ \mathbf { ~ }}$ & $\mathbf{1 2 2 3} \mathbf{K}$ & $\mathbf{1 2 7 3 ~ \mathbf { ~ }}$ \\
\cline { 2 - 6 } & 23.9 & 23.9 & 23.9 & 23.9 & 23.9 \\
\hline 0 & 25.8 & 26.0 & 26.6 & 28.5 & 28.4 \\
\hline 2 & 27.5 & 28.5 & 31.6 & 31.6 & 32.8 \\
\hline 5 & 29.2 & 32.1 & 35.5 & 34.2 & 36.4 \\
\hline 9 & & & & &
\end{tabular}

Figure 3 shows reduction behaviors of $\mathrm{FeO}$ for various temperatures ranging from 1073-1273 K. As the temperature increases, the reduction rate and the final reduction degree increase simultaneously in general. There are typical results based on the fundamental laws of diffusion theory and basic principles of chemical thermodynamics. El-Geassy [25] has reported that the activation energy is $133.97 \mathrm{~kJ} /$ mole when pure FeO is reduced, and the activation energy based on the results of this experiment shows a similar value of $137.78 \mathrm{~kJ} /$ mole.

Figure 4 shows reduction behaviors of $\mathrm{FeO}$ with various porosities in the temperature range of 1073-1273 K. Despite the increase in overall porosity by introducing extrinsic pores, the initial reduction rate abnormally tends to decrease. The overall porosity and initial reduction rate followed an inverse relationship, and the trend shows an inflection point at which the reduction rate increases after approximately $40 \%$ of the degree of the reduction process. The tendency to increase the reduction rate after $40 \%$ reduction degree increased with the extrinsic pores. In addition, this abnormal behavior related to pores gradually decreased with increasing temperature. Bahgat [19] and Kim [20] reported that the initial porosity and reduction rate are proportional to each other. The positive effect of porosity on reduction rate is generally accepted. However, the results of this experiment show a behavior contrary to the general view of porosity and reduction rate. 


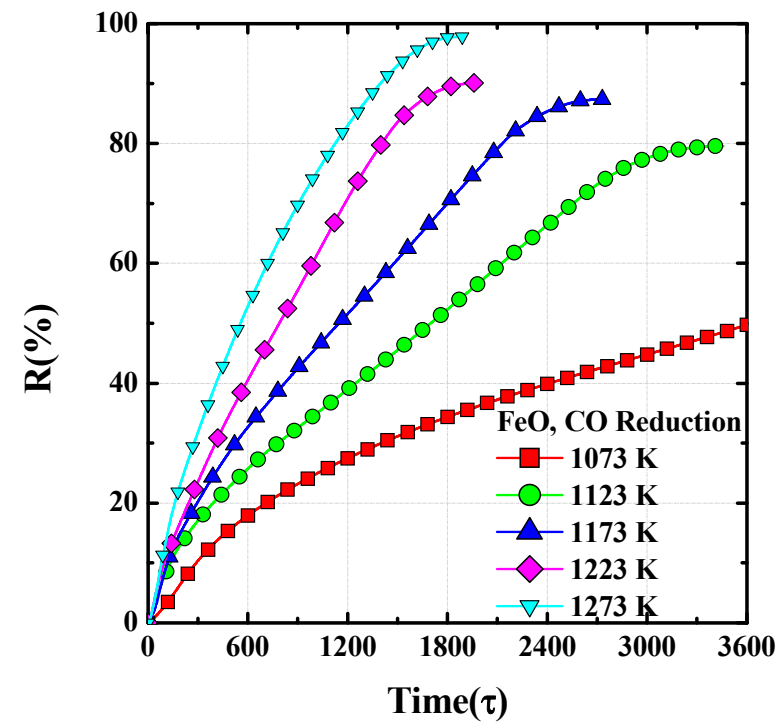

Figure 3. Reduction behaviors of $\mathrm{FeO}$ at various temperatures in the range of 1073-1273 K (time $\tau$ : reset the time when reduction starts).

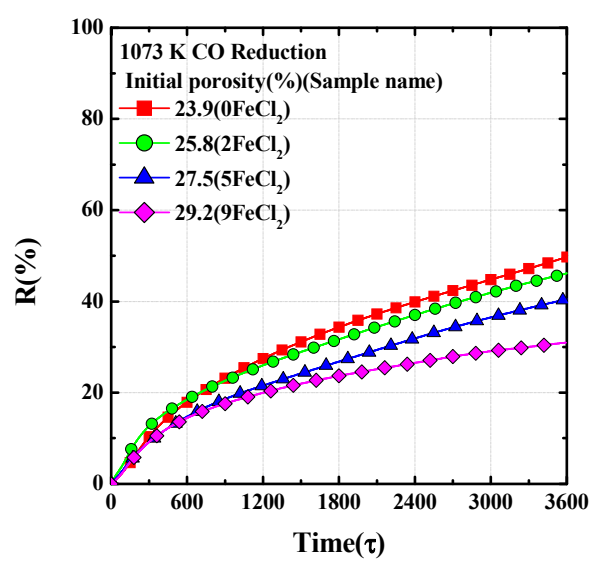

(a)

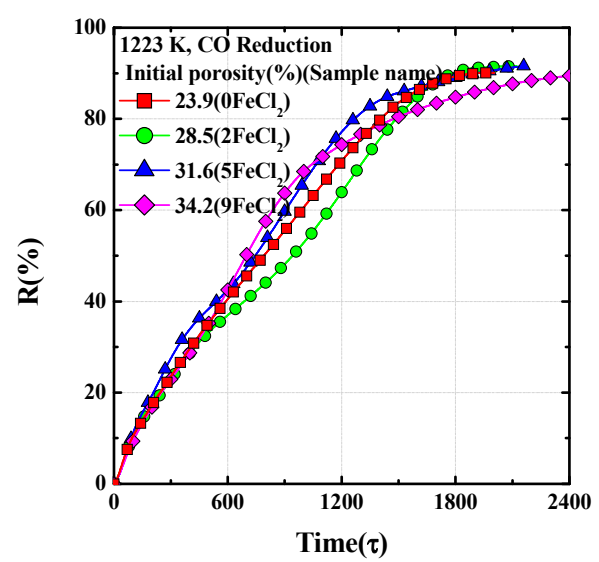

(d)

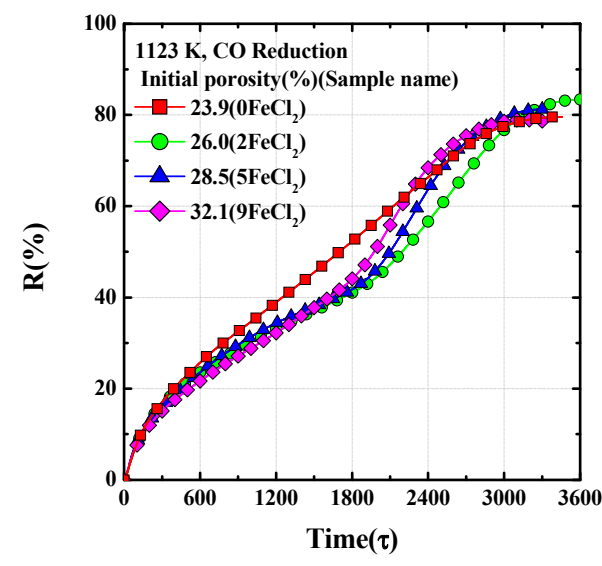

(b)

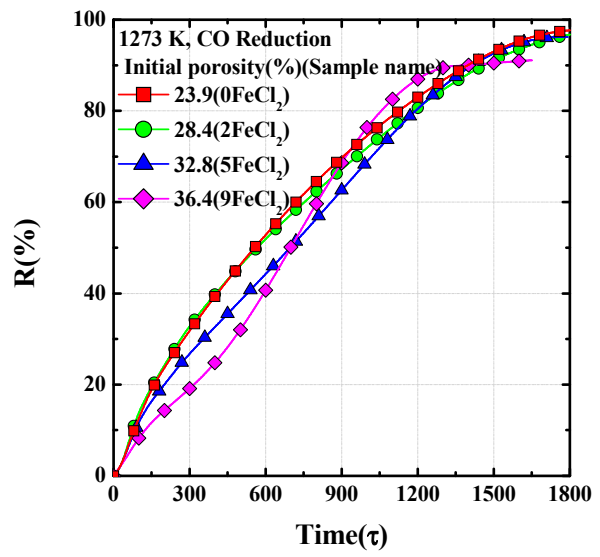

(e)

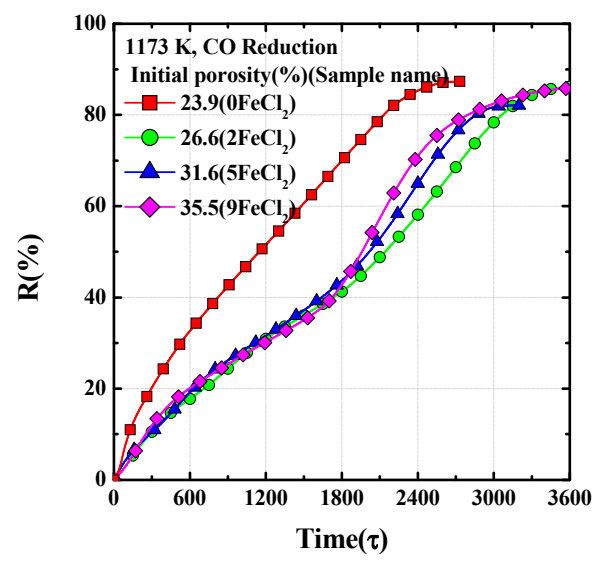

(c)

Figure 4. Reduction behaviors of FeO with various porosities in the temperature range of 1073-1273 K; (a) $1073 \mathrm{~K}$, (b) $1123 \mathrm{~K}$, (c) $1173 \mathrm{~K},(\mathbf{d}) 1223 \mathrm{~K}$, and (e) $1273 \mathrm{~K}$ (time $\tau$ : reset the time when reduction starts). 
The experimental results of this experiment are mathematically analyzed using the grain model proposed by Szekely [26]. In the heterogeneous gas-solid reaction model of the cylindrical compact, the interfacial chemical reaction can be expressed as follows:

$$
g_{F_{g}}(X)=1-(1-X)^{\frac{1}{2}}=k_{I C R} \cdot \tau
$$

The gaseous mass transport through the product layer can be expressed as:

$$
p_{F_{P}}=X+(1-X) \ln (1-X)=k_{G M T} \cdot \tau .
$$

The mixed control can be expressed as follows:

$$
g_{F_{g}}+p_{F_{P}}=k_{\text {Mixed }} \cdot \tau,
$$

where $g_{F_{g}}$ and $p_{F_{g}}$ are the conversion functions and $X$ is the fraction of reduction at a given time $(\tau) . k_{I C R}, k_{G M T}$, and $k_{\text {Mixed }}$ are the apparent rate constants of the interfacial chemical reaction, gaseous mass transport through the product layer, and mixed control, respectively. If the correlation of time ( $\tau$ ) in Equations (2)-(4) shows a linear relationship, the rate constant can be derived from the slope of the straight line. The results of applying the formulas to the $\mathrm{FeO}$ reduction are presented in Figure $5 . k_{\text {Mixed }}^{\mathrm{FeO} \text { introduced pore }}$ and $k_{\text {Mixed }}^{\text {pure }}$ are the apparent rate constants in the mixed control of $\mathrm{FeO}$ introduced by the extrinsic pore and pure $\mathrm{FeO}$. At the initial stage of reduction, the reduction rate decreases in proportion to the extrinsic pores. In addition, the dependence of the reduction rate on the extrinsic pores is relaxed by increasing the temperature. At a later stage of reduction (after the inflection point), the relationship between the reduction rate and extrinsic pores reverted to a normal behavior.

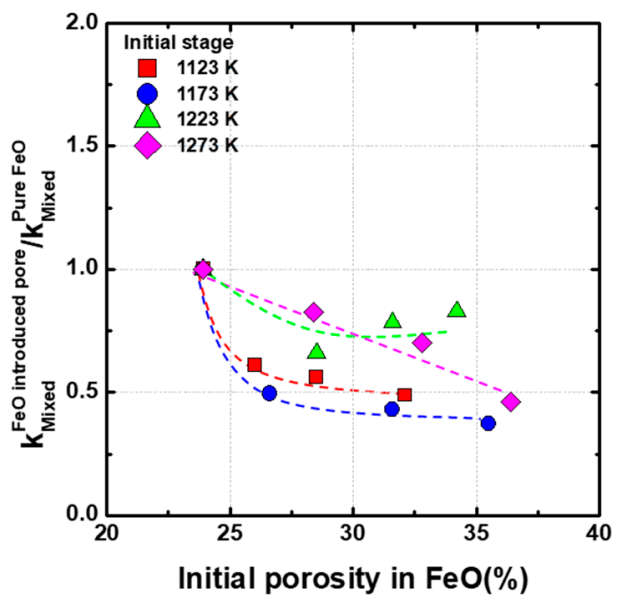

(a)

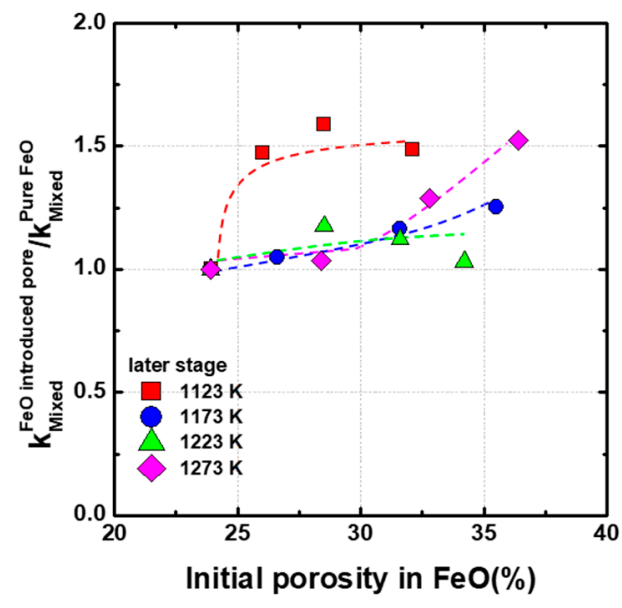

(b)

Figure 5. Ratios of apparent reduction rate constants of extrinsic pore introduced FeO and pure FeO for each initial overall porosity in FeO. (a) Initial stage, (b) Later stage

The dimensionless kinetic parameter, $\mathrm{k}_{\text {Later stage }} / \mathrm{k}_{\text {Initial stage, }}$ is shown in Figure 6, where $\mathrm{k}_{\text {Later stage }}$ and $\mathrm{k}_{\text {Initial stage }}$ represent the apparent rate constants in the initial and later stages, respectively. The rate constants of gaseous mass transport through the product layer against the interfacial chemical reaction significantly increased after half of the reduction is complete, compared to those observed for the initial reduction; further, the gaseous mass transport rate constants increased by 2-7 times after the later stage reduction. The abnormal reduction behavior is more affected by the diffusion of gas than by the chemical reaction. In addition, at $1173 \mathrm{~K}$, where abnormal behavior is well confirmed, Figure 7 shows the apparent gaseous rate constant at the initial and later stages. In the initial stage of 
reduction, although the initial overall porosity in $\mathrm{FeO}$ increases, the apparent gaseous rate constant tends to decrease. However, in the later reduction stage, the apparent gaseous rate constant tends to increase in proportion to the initial overall porosity. It is believed that the diffusion behavior of the reducing gas changes because of the change in the morphology of $\mathrm{FeO}$ during the reduction process.

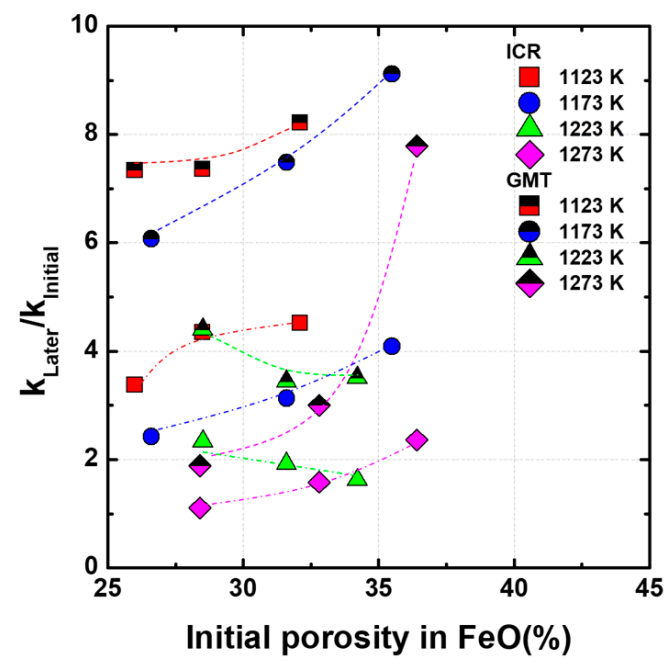

Figure 6. Ratios of apparent reduction rate constants of initial reduction stage and later reduction stage for each initial overall porosity in FeO.

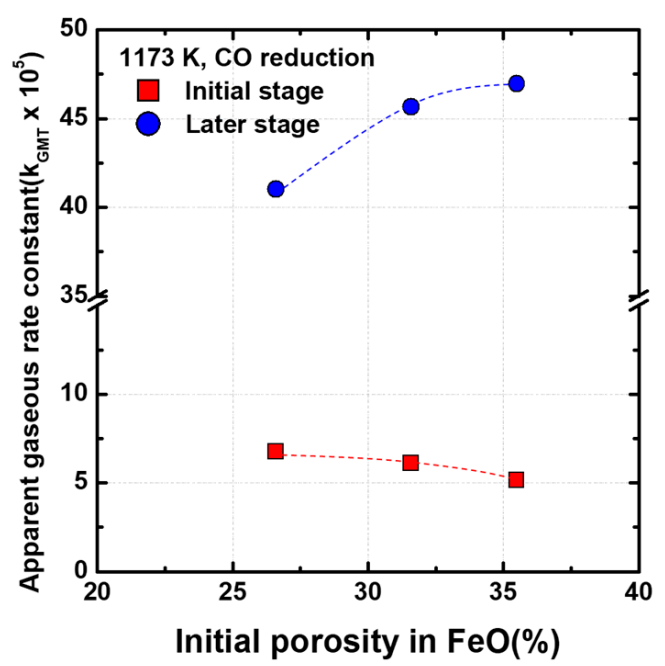

Figure 7. Apparent gaseous rate constant for each initial overall porosity in $\mathrm{FeO}$ at $1173 \mathrm{~K}$.

To confirm the morphology of the sample before reduction at $1173 \mathrm{~K}$, the sample was analyzed using SEM and the results are shown in Figure 1. In the case of pure $\mathrm{FeO}$ (Figure 1a), it has spherical morphology. On the other hand, in the case of $\mathrm{FeO}$ with extrinsic pores (Figure 1d), the spherical morphology mostly changed to a large plate-like morphology. In the case of increasing extrinsic pores in $\mathrm{FeO}$, the plate-like morphology becomes pronounced. The experimental results indicate that the quality of the extrinsic pores plays an important role in controlling the initial reduction rate. Therefore, it is necessary to examine the dependency of not only the number of pores, but also other aspects of the relationship between the pores and the reduction rate.

The relationship between the characteristics of pores and the reducing gas permeability can be evaluated using the labyrinth coefficient. The labyrinth coefficient represents the 
relationship between pore quantity and tortuosity, and it can be used to evaluate the gas permeability through the pores. The labyrinth coefficient can be expressed as follows [27]:

$$
D_{s}=\frac{1+K}{K} \cdot \frac{K D_{\mathrm{CO}}^{(P)} D_{\mathrm{CO}_{2}}^{(P)}}{D_{\mathrm{CO}}^{(P)}+K D_{\mathrm{CO}_{2}}^{(P)}} \varepsilon \cdot \xi,
$$

where $\xi$ is the labyrinth coefficient, $\varepsilon$ is the porosity of the $\mathrm{FeO}$ pellet, $\mathrm{K}$ is the equilibrium constant, $D_{\mathrm{CO}}^{(P)}$ and $D_{\mathrm{CO}_{2}}^{(P)}$ are the diffusion coefficients of $\mathrm{CO}$ and $\mathrm{CO}_{2}$ in the pore. In addition, DS is an effective diffusion coefficient that can be calculated by Ishida-Wen's model [28]. A labyrinth coefficient value indicates the gas permeability in the FeO pellet. Applying this experimental data to Equation (5), it is shown in Figure 8 as a function of the initial porosity in $\mathrm{FeO}$. The initial reduction stage of porosity in $\mathrm{FeO}$ increases, and the labyrinth coefficient tends to decrease. This means that the extrinsic pores mainly generated in $\mathrm{FeO}$ by the evaporation of $\mathrm{FeCl}_{2}$ are closed or open only at one end, which have a negative effect on reduction. Therefore, despite the increase in the overall pores, the initial reduction rate decreases because the formed pores are not suitable for reduction. However, as the reduction proceeds, the porosity in $\mathrm{FeO}$ increases and the labyrinth coefficient also tends to increase. During the reduction process, the structure of morphology changed in favor of gas diffusion, thereby increasing the labyrinth coefficient. These results are in good agreement with Kamijo's [29] research. Accordingly, it is thought that reduction behavior exhibits an abnormal behavior that accelerates the reduction rate after half of the reduction process is complete.

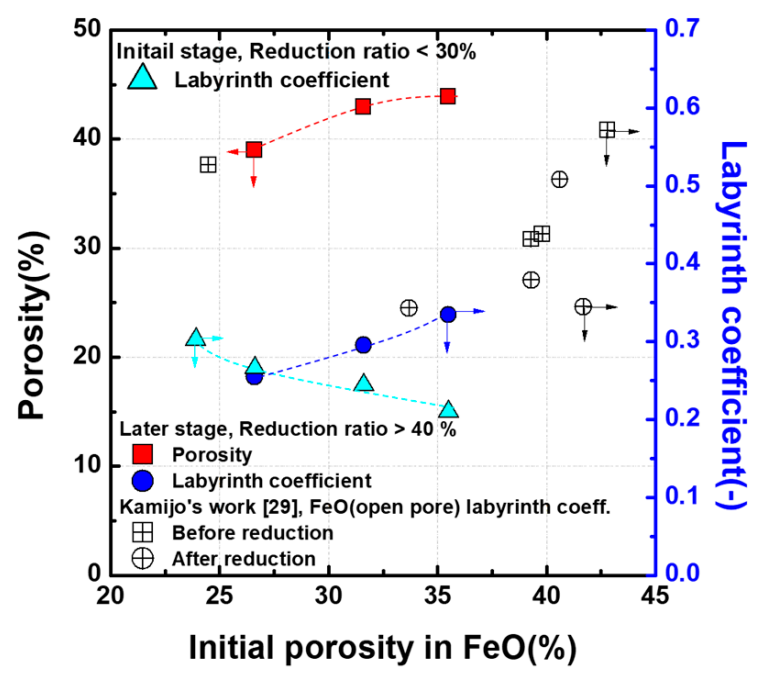

Figure 8. The labyrinth coefficient of the initial stage and the labyrinth coefficient and porosity of the later stage according to the initial overall porosity in $\mathrm{FeO}$, obtained at $1173 \mathrm{~K}$.

Figure 9 shows the effect of the initial overall porosity on the morphological change of reduced iron at $1173 \mathrm{~K}$. For pure $\mathrm{FeO}$ with an initial porosity of $23.9 \%$, spherical particles can be seen at the beginning of the reduction process, and the morphology changes into the porous state as the reduction progresses. With increasing initial overall porosity, a large plate-like morphology $\mathrm{FeO}$ can be seen at the beginning of the reduction process. In the initial stage of reduction, the diffusion of the reducing gas decreases due to the large plate-like morphology of $\mathrm{FeO}$ and the labyrinth coefficient decreases. Because of the difference in density between $\mathrm{FeO}$ and reduced iron, the large plate-like morphology $\mathrm{FeO}$ becomes smaller with the reduction procedure, and pores that are open only at one end evolve into those that are open at the two ends. Owing to the change in the pore shape, the labyrinth coefficient increases in proportion to the porosity in $\mathrm{FeO}$ after half the reduction process is complete. As a result, the flow of the reducing gas becomes smooth, and the 
reduction rate increases after half of the reduction process is carried out. After complete reduction, all the samples showed porous morphology, even though the initial porosity changed by the introduction of $\mathrm{FeCl}_{2}$.

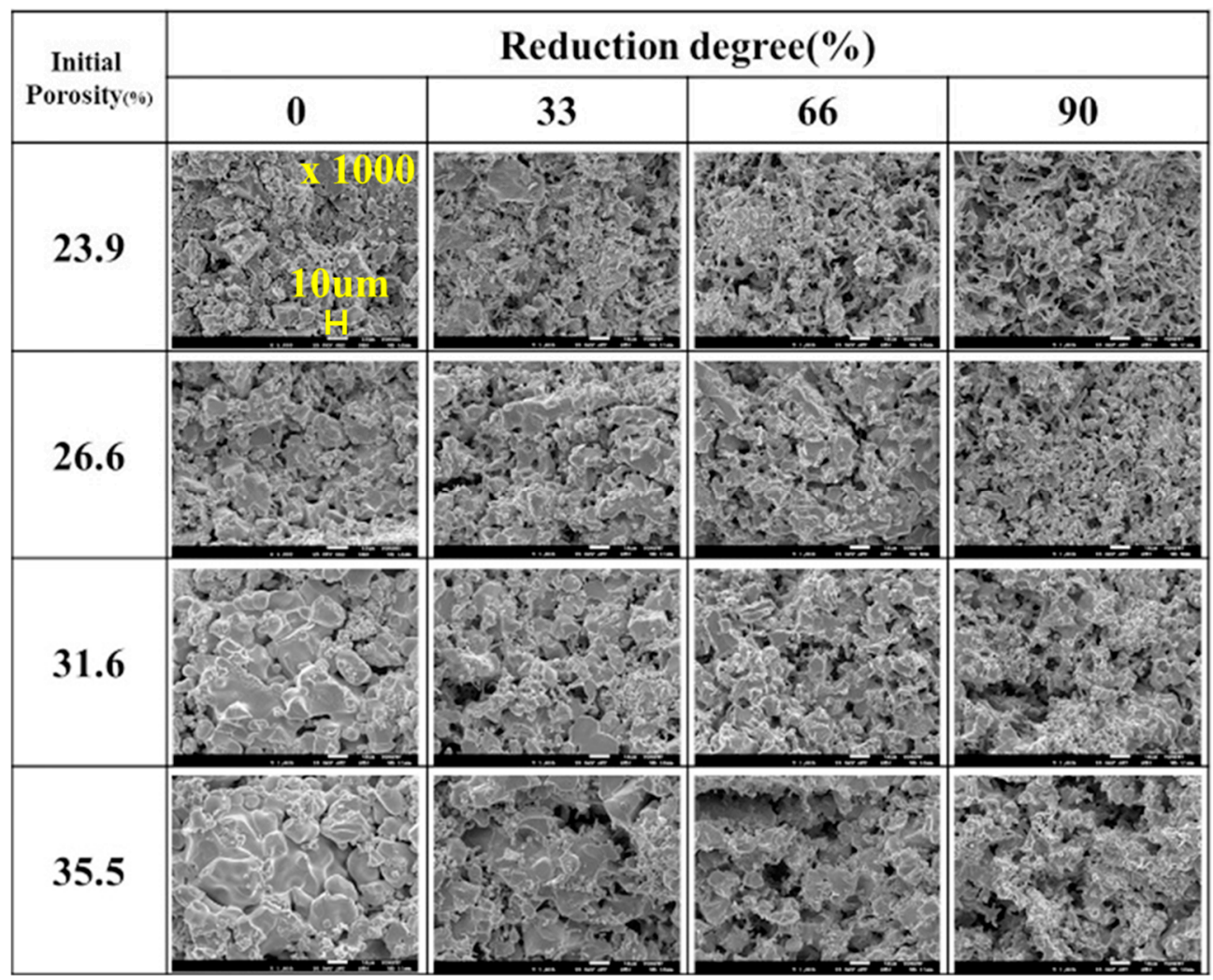

Figure 9. Morphological changes of $\mathrm{FeO}$ samples at $1173 \mathrm{~K}$ at different degrees of reduction and initial overall porosity in FeO.

The effect of temperature on the morphology of FeO is shown in Figure 10. At $1073 \mathrm{~K}$, $\mathrm{FeO}$ showed large plate-like morphology. However, as the temperature increases, $\mathrm{FeO}$ has a morphology similar to that of pure $\mathrm{FeO}$ (spherical morphology). Therefore, as the temperature increases, the initial reduction rate of $\mathrm{FeO}$ that formed pores because of the introduction of $\mathrm{FeCl}_{2}$ is similar to that of pure $\mathrm{FeO}$. However, even at high temperatures, because of the formation of pores that are open only at one end having a large plate-like morphology, the initial reduction rate appears to decrease.

The formation of extrinsic pores of the open only at one end by $\mathrm{FeCl}_{2}$ addition, and their behavior during reduction process is schematically represented in Figure 11. In the heating stage (Figure 11a), at a relatively low temperature (1173 K), $\mathrm{FeCl}_{2}$ forms a liquid phase with $\mathrm{FeO}$ and then evaporates. As a result, several pores that are open only at one end are formed. However, at a relatively high temperature (1273 K), many fractions of $\mathrm{FeCl}_{2}$ evaporate without reacting with $\mathrm{FeO}$. As a result, fewer number of pores open only at one end are produced at lower temperatures. In the reduction stage (Figure 11b), at relatively low temperatures $(1173 \mathrm{~K})$, the reduction rate decreases because of the formation of pores that are open only at one end, despite the increase in the overall porosity of the sample. During the reduction process, $\mathrm{FeO}$ with a large plate-like morphology is reduced to porous $\mathrm{Fe}$, leading to the disappearance of pores open only at one end. As a result, the reduction rate increases after the middle stage of reduction. At a relatively high temperature (1273 K), the reduction behavior is similar to that at lower temperatures. However, since the number of open only at one end pore is less, the tendency of unusual behavior at low temperatures is low. After completion of the reduction process, porous iron with similar morphology is formed at both high and low temperatures. 

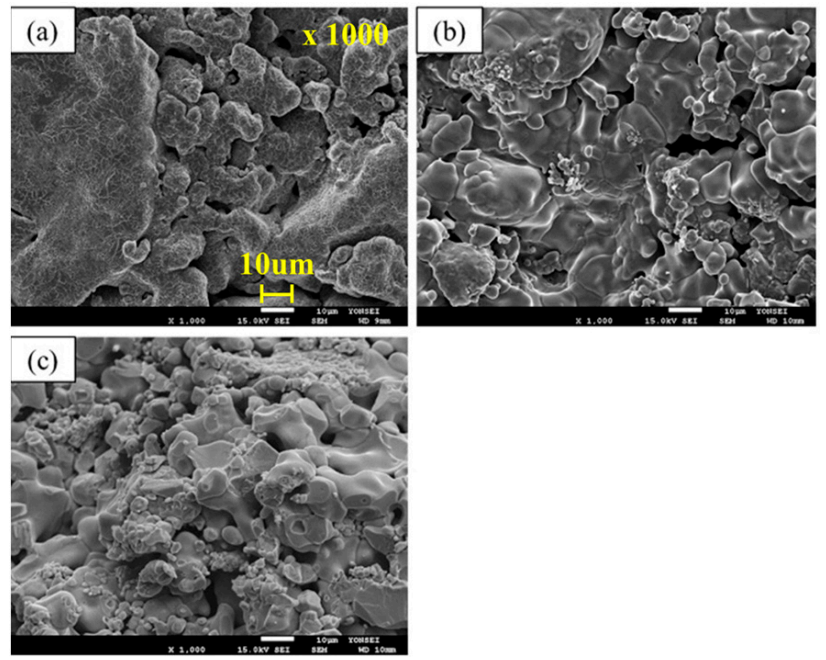

Figure 10. SEM micrographs of the FeO sample at different temperatures, obtained using $9 \mathrm{FeCl}_{2}$ sample under the condition of the inert gas; (a) $1073 \mathrm{~K}$, (b) $1173 \mathrm{~K}$, and (c) $1273 \mathrm{~K}$.

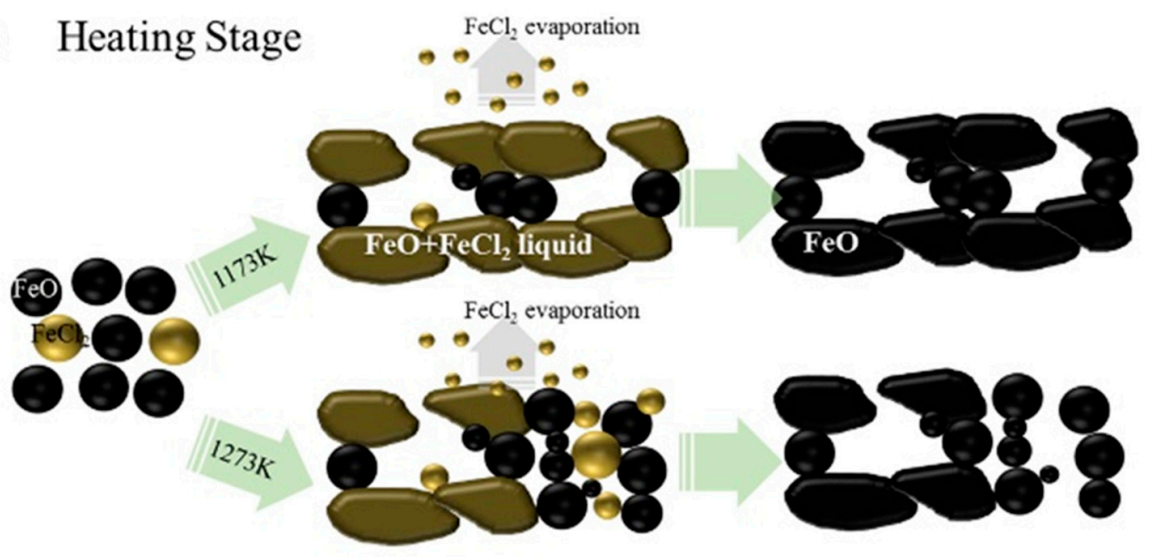

(a)

Reduction Stage
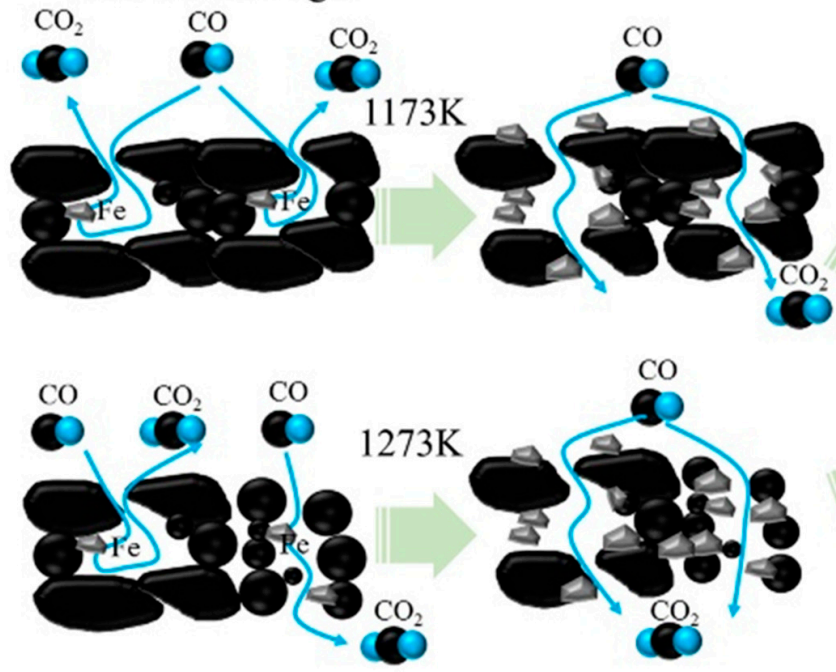

Reduction completed

(b)

Figure 11. Schematic for conceptual morphological evolution of $\mathrm{FeO}-\mathrm{FeCl}_{2} ;(\mathbf{a})$ heating stage and (b) reduction stage. 
It is generally accepted that the reduction rate of iron oxide is proportional to the overall porosity [16-21]. However, if the sample has pores open only at one end or closed pores, the reduction rate is not proportional to the overall porosity. Therefore, while considering the pores for the reduction rate, the number of pores as well as type and distribution of pores should be included.

\section{Conclusions}

To quantify the effect of pores on the reduction rate, pores were artificially created in $\mathrm{FeO}$ using the $\mathrm{FeCl}_{2}$ evaporation technique. Extrinsic pores were successfully created in $\mathrm{FeO}$ using $\mathrm{FeCl}_{2}$. However, the initial reduction rate tended to be inversely proportional to the overall porosity, and the behavior of the reduction rate increased after half of the reduction process was complete. The abnormal reduction behavior was confirmed through mathematical modeling, labyrinth coefficient, and SEM analysis. Extrinsic pores open only at one end were formed and observed to be directly proportional to the initial content of $\mathrm{FeCl}_{2}$. Although the overall porosity of the sample increased, the initial reduction rate decreased because of the pores that are open only at one end. As the reduction progresses, the pores open only at one end disappear and the reduction rate increases after half the reduction process. The reduction rate and overall porosity tend to be proportional to each other. The pores formed at the initial stage of the reduction are retained until the end of the process. In addition, it is confirmed that not only the number of pores, but also the characteristics of porosity are important while considering the effect of pores on the reduction rate.

Author Contributions: Writing—original draft preparation, S.G.S.; Writing—rewiew and editing, D.J.M. Both authors have read and agreed to the published version of the manuscript.

Funding: This research received no external funding.

Institutional Review Board Statement: Not applicable.

Informed Consent Statement: Not applicable.

Data Availability Statement: Data sharing not applicable.

Acknowledgments: This work was supported by the Korea Institute of Energy Technology Evaluation and Planning (KETEP) and the Ministry of Trade, Industry \& Energy (MOTIE) of the Republic of Korea (No. 20172010106300).

Conflicts of Interest: The authors declare no conflict of interest.

\section{References}

1. Turkdogan, E.T.; Vinter, J.V. Gaseous reduction of iron oxides: Part III. Reduction-oxidation of porous and dense iron oxide and iron. Metall. Trans. 1972, 3, 1561-1574. [CrossRef]

2. El-Geassy, A.A.; Rajakumar, V. Gaseous Reduction of Wustite with H2, CO and H2-CO Mixture. Trans. ISIJ 1985, $25,449-458$. [CrossRef]

3. El-Geassy, A.A. Gaseous reduction of Fe2O3 compacts at 600 to $1050{ }^{\circ}$ C. J. Mater. Sci. 1986, 21, 3889-3900. [CrossRef]

4. Piotrowski, K.; Mondal, K.; Lorethova, H.; Stonawski, L.; Sztmanski, T.; Wiltowski, T. Effect of gas composition on the kinetic of iron oxide reduction in a hydrogen production process. Int. J. Hydrogen Energy 2005, 30, 1543-1554. [CrossRef]

5. Olsson, R.G.; Mckewan, W.M. Diffusion of H2-H2O through porous iron formed by the reduction of iron oxides. Metall. Trans. 1970, 1, 1507-1512. [CrossRef]

6. Bustnes, J.A. Kinetic studies of the reduction of FeO and FeWO4 by hydrogen. Metall. Mater. Trans. B 1997, $28 B, 613-618$. [CrossRef]

7. Pineau, A.; Kanari, N.; Gaballah, I. Kinetics of reduction of iron oxides by H2 part I: Low temperature reduction of hematite. Thermochim. Acta 2006, 447, 89-100. [CrossRef]

8. Pineau, A.; Kanari, N.; Gaballah, I. Kinetics of reduction of iron oxides by H2 part II: Low temperature reduction of magnetite. Thermochim. Acta 2007, 456, 75-88. [CrossRef]

9. Yamashita, T.; Nakada, T.; Nagata, K. In-situ observation of $\mathrm{Fe}_{0.94} \mathrm{O}$ reduction at high temperature with the use of optical microscopy. Metall. Mater. Trans. B 2007, 38B, 185-191.

10. El-geassy, A.A. Gaseous reduction of MgO-doped Fe2O3 compacts with carbon-monoxide at 1173-1473 K. ISIJ Int. 1996, 36, 1328-1337. [CrossRef] 
11. El-geassy, A.A. Influence of doping with $\mathrm{CaO}$ and/or $\mathrm{MgO}$ on stepwise reduction of pure hematite compacts. Ironmak. Steelmak. 1999, 26, 41-52. [CrossRef]

12. Paananen, T.; Heinanen, K.; Harikki, J. Degradation of iron oxide caused by alumina during reduction from magnetite. ISIJ Int. 2003, 43, 597-605. [CrossRef]

13. Inami, T.; Suzuki, K. Effect of $\mathrm{SiO} 2$ and $\mathrm{Al} 2 \mathrm{O} 3$ on the lattice parameter and $\mathrm{CO}$ gas reduction of CaO-containing dense wustite. ISIJ Int. 2003, 43, 314-320. [CrossRef]

14. Scarlett, N.V.Y.; Pownceby, M.I.; Madsen, I.C.; Christensen, A.N. Reaction sequences in the formation of silico-ferrites of calcium and aluminum in iron ore sinter. Metall. Mater. Trans. B 2004, 35B, 929-936. [CrossRef]

15. El-geassy, A.A.; Nasr, M.I.; Omar, A.A.; Mousa, E.A. Influence of $\mathrm{SiO} 2$ and/or $\mathrm{MnO} 2$ on the reduction behavior and structure changes of Fe2O3 compacts with CO gas. ISIJ Int. 2008, 48, 1359-1367. [CrossRef]

16. Huang, Z.; Yi, L.; Jiang, T. Mechanisms of strength decrease in the initial reduction of iron ore oxide pellets. Powder Technol. 2012, 221, 284-291. [CrossRef]

17. Turkdogan, E.T.; Olsson, R.G.; Vinters, J.V. Gaseous reduction of iron oxides: Part II. Pore characteristic of iron reduced from hematite in hydrogen. Metall. Trans. 1971, 2, 3189-3196. [CrossRef]

18. Matthew, S.P.; Cho, T.R.; Hayes, P.C. Mechanisms of porous iron growth on wustite and magnetite during gaseous reduction. Metall. Trans. B 1990, 21B, 733-741. [CrossRef]

19. Bahgat, M.; Halim, K.S.; Nasr, M.I.; El-geassy, A.A. Morphological changes accompanying gaseous reduction of SiO2 doped wustite compacts. Ironmak. Steelmak. 2008, 35, 205-212. [CrossRef]

20. Kim, W.H.; Lee, Y.S.; Suh, I.K.; Min, D.J. Influence of $\mathrm{CaO}$ and SiO2 on the reducibility of wustite using H2 and CO gas. ISIJ Int. 2012, 52, 1463-1471. [CrossRef]

21. Takahashi, K.; Asada, M.; Kawakami, M. TEM observation MgO- or CaO-bearing wustite solid solution reduced to iron by hydrogen. Tetsu Hagane 1998, 84, 471-476. [CrossRef]

22. Kaneko, K. Determination of pore size and pore size distribution 1. Adsorbents and catalysts. J. Membrane Sci. 1994, 96, 54-89. [CrossRef]

23. Rouquerol, J.; Avnir, D.; Fairbridge, C.W.; Everett, D.H.; Haynes, J.H.; Pernicone, N.; Ramsay, J.D.F.; Sing, K.S.W.; Unger, K.K. Recommendations for the characterization of porous solids. Pure Appl. Chem. 1994, 66, 1739-1758. [CrossRef]

24. Kubaschewski, O.; Alcock, C.B. Metallurgical Thermochemistry, 5th ed.; Pergamon Press: Oxford, UK, 1979 ; p. 362.

25. El-geassy, A.A. Rate controlling step in the reduction of iron oxides; Kinetics and mechanism of wustite-iron step in $\mathrm{H} 2, \mathrm{CO}$ and H2/CO gas mixtures. IOP Conf. 2017, 229, 1-10. [CrossRef]

26. Szeckely, J.; Evans, J.W.; Sohn, H.Y. Gas-Solid Reaction; Academic Press: New York, NY, USA, 1976; pp. $125-147$.

27. Yagi, T.; Ono, Y. A method of analysis for reduction of iron oxide in mixed-control kinetics. ISIJ Int. 1968, 8, 377-381. [CrossRef]

28. Murayama, T.; Ono, Y. Method of determination of parameters included in Ishida-Wen's model. Tetsu Hagane 1987, 10, 1323-1328. [CrossRef]

29. Kamijo, C. Method of estimation of reduction rate constant in Ishida-Wen's model for FeO-Al2O3 briquette. ISIJ Int. 2017, 57, 1797-1803. [CrossRef] 